\title{
Hemisorption of hydrogen on the diamond surface containing a "boron + vacancy" defect
}

\author{
O. Yu. Ananina ${ }^{1}$, O. V. Ponomarev ${ }^{2}$, A. I. Ryazanova ${ }^{2,3}$, N. A. Lvova ${ }^{2,3}$ \\ ${ }^{1}$ Physical Faculty, Zaporizhzhya National University, Zaporizhzhya, Ukraine \\ ${ }^{2}$ Technological Institute for Superhard and Novel Carbon Materials, Troitsk, Moscow, Russia \\ ${ }^{3}$ Moscow Institute of Physics and Technology, Dolgoprudny, Moscow Region, Russia \\ ananyina@znu.edu.ua, oleg.ponomarev@phystech.edu, ryazanova@phystech.edu,nlvova@tisnum.ru
}

PACS 68.43.-h, 81.05.ug, 03.67.Lx

DOI 10.17586/2220-8054-2018-9-1-61-63

\begin{abstract}
In this paper, we present the results of quantum-chemical modeling for atomic hydrogen adsorption on the $\mathrm{C}(100)-(2 \times 1)$ diamond surface containing a "boron + monovacancy" complex defect. We also provide a comparison of the energy characteristics of adsorption (activation energy and adsorption heat) for an ordered diamond surface, graphene surface, and a surface containing a "boron + monovacancy" complex defect.
\end{abstract}

Keywords: C(100)-(2×1) diamond surface, quantum-chemical modeling, complex defect, BV-complex, adsorption.

Received: 19 June 2017

Revised: 18 October 2017

\section{Introduction}

Boron-doped diamond is a $p$-type semiconductor and due to its unique natural properties, it is a promising material for microelectronics [1]. Goss and coworkers studied boron aggregates in diamond [2]. According to $a b$ initio calculations, the $\mathrm{B}_{4} \mathrm{~V}$ - and $\mathrm{BV}$-complexes correspond to the lowest energy of formation attributed to a single $\mathrm{B}$ atom. In [3], the authors study the electronic structure of a BV-center in diamond for its various charge states $-\mathrm{BV}^{0}, \mathrm{BV}^{+1}, \mathrm{BV}^{-1}$ and $\mathrm{BV}^{-2}$ - using first-principles calculations. The charge state of the $\mathrm{BV}^{-1}$ complex defect is stable and is suitable for qubit realization. However, for the reconstructed $C(100)-(2 \times 1)$ surface, the BV-complexes remain insufficiently studied. Such a complex defect located on the surface or in the near-surface layers can lead to local surface restructuring, to the charge distribution between the atoms around the defect, and can influence the energy of particle adsorption. Atomic hydrogen takes part in the growth mechanisms of CVD diamonds [4], in diamond etching for use in microelectronics [5].

In this paper, we investigate the energy characteristics of hydrogen adsorption on a $\mathrm{C}(100)-(2 \times 1)$ diamond surface with BV-complexes by quantum-chemistry methods.

\section{Calculation technique}

A clean reconstructed $\mathrm{C}(100)-(2 \times 1)$ diamond surface was reproduced with use of $\mathrm{C}_{195} \mathrm{H}_{112}$ and $\mathrm{C}_{198} \mathrm{H}_{100}$ cluster models containing 5 and 8 atomic layers, respectively. Calculations involved semi-empirical quantumchemical methods implemented in the MOPAC software package [6]. The distance between the hydrogen atom and the surface carbon atom was chosen as the reaction coordinate for calculation of $\mathrm{H}$ adsorption. The calculation procedure is described in detail in [7].

\section{Adsorption of atomic hydrogen on the diamond surface with a BV-complex}

Calculations carried out for the cluster surface models have shown [8] that the lowest energy belongs to the state of a complex defect in which boron is in the fourth layer, and the vacancy is in the third layer (Fig. 1, defect 1). The position of boron in the first layer, with the same position of the vacancy in the third layer, is less energetically favorable (Fig. 1, defect 2). The feature of defect 1 is the formation of C1-C6 hexagonal structure on the surface [8]. The order of bonds between the "hexagon" atoms differs significantly from the order of these atomic bonds on the ordered surface and is close to the bond order of carbon atoms in graphene (Table 1). Two types of adsorption centers can be distinguished among atoms of the "hexagon" on the surface: they are C1-C2 and $\mathrm{C} 3-\mathrm{C} 4$ atoms (initially, atoms of the surface dimers) and C5 and C6 atoms (initially, the second-layer atoms). In the structure of defect 2, replacing the C5 carbon atom by the B boron atom resulted in distortion of the "graphene-like" structure: all sides of the "hexagon" have different lengths. The most bound pairs of atoms are $\mathrm{C} 1-\mathrm{C} 6$ and $\mathrm{C} 4-\mathrm{C} 3$. 


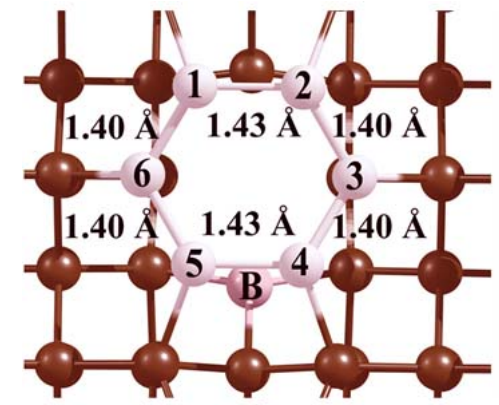

a)

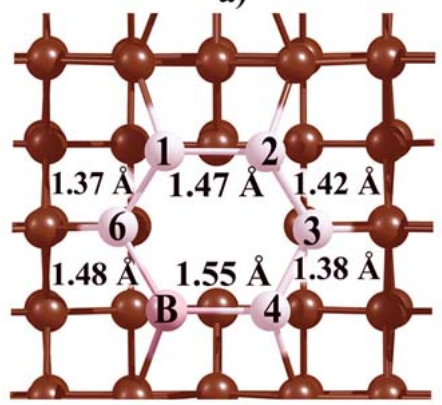

d)

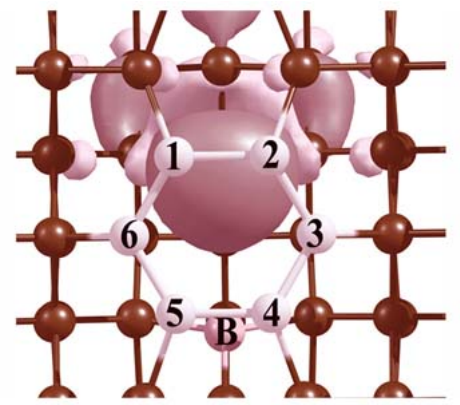

b)

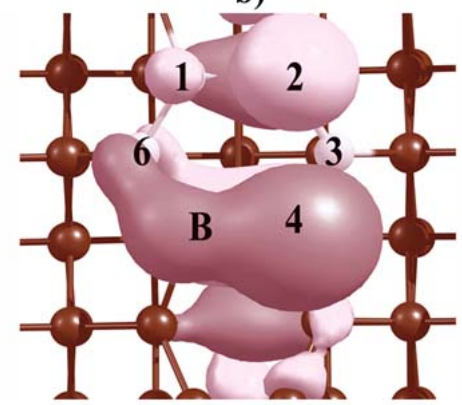

e)

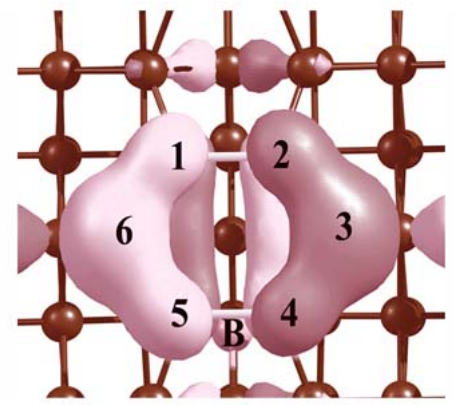

c)

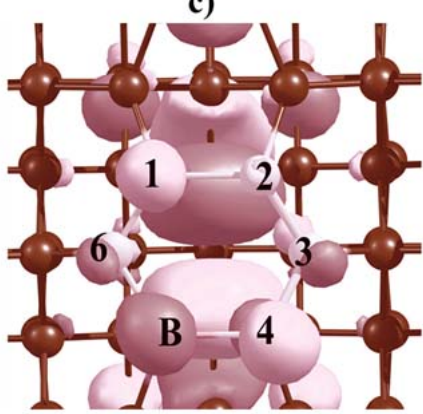

f)

FIG. 1. a), d) defect 1 and defect 2; b), e) HOMO electron density isosurfaces; c) isosurface of the electron density of MO localized on the atoms of the "hexagon"; f) LUMO electron density isosurfaces. Atoms of the "hexagon" are shown in light gray color

TABLE 1. Bond orders (b.o)and interatomic distances $(d)$ of carbon atoms on the $\mathrm{C}(100)-(2 \times 1)$ diamond surface containing a "boron+vacancy" complex, and in graphene

\begin{tabular}{|c|c|c|c|c|c|c|c|c|}
\hline \multirow[t]{2}{*}{ Atoms } & \multicolumn{2}{|c|}{ Ordered surface } & \multicolumn{2}{|c|}{ Defect 1} & \multicolumn{2}{|c|}{ Defect 2} & \multicolumn{2}{|c|}{ Graphene $[9,10]$} \\
\hline & b.o & $d, \AA$ & b.o & $d, \AA$ & b.o & $d, \AA$ & b.o & $d, \AA$ \\
\hline $\mathrm{C} 1-\mathrm{C} 2$ & 1.85 & 1.400 & 1.35 & 1.430 & 1.15 & 1.472 & 1.32 & \multirow{6}{*}{1.420} \\
\hline $\mathrm{C} 2-\mathrm{C} 3$ & 0.96 & 1.508 & 1.39 & 1.402 & 1.29 & 1.421 & 1.32 & \\
\hline C3-C4 & 0.96 & 1.508 & 1.39 & 1.401 & 1.53 & 1.383 & 1.32 & \\
\hline $\mathrm{C} 5(\mathrm{~B})-\mathrm{C} 4$ & 1.85 & 1.400 & 1.35 & 1.431 & 1.04 & 1.550 & 1.32 & \\
\hline $\mathrm{C} 5(\mathrm{~B})-\mathrm{C} 6$ & 0.96 & 1.508 & 1.38 & 1.401 & 1.13 & 1.482 & 1.32 & \\
\hline $\mathrm{C} 1-\mathrm{C} 6$ & 0.96 & 1.508 & 1.39 & 1.403 & 1.60 & 1.371 & 1.32 & \\
\hline
\end{tabular}

The energy of the dimeric $\pi$-bond on the C(100)- $(2 \times 1)$ surface was estimated by ab initio methods in [11], and for different cluster models it was $0.67-1.17 \mathrm{eV}$. The authors of [12] estimated the activation energy of atomic hydrogen chemisorption on the surface dimer atoms as $E_{a c t}=0.65 \mathrm{eV}$ using semiempirical quantum chemistry methods. Comparison of the $E_{a c t}=0.85-1.11 \mathrm{eV}$ values obtained in this work for a hexagonal graphene-like structure on the surface with a BV-complex (defect 1) with the data of [11,12] suggests that the chemisorption of hydrogen will primarily occur on the ordered areas of the surface where surface dimer atoms are the adsorption centers $\left(E_{a c t}=0.65 \mathrm{eV}\right)$, and only then on hexagon atoms - predominantly on the $\mathrm{C} 1, \mathrm{C} 2, \mathrm{C} 4$ and $\mathrm{C} 5$ atoms, for which the activation energy of the first hydrogen atom adsorption is $E_{a c t}=0.85 \mathrm{eV}$ (Table 2).

All hexagon atoms in defect 2 can be called adsorption centers of different types; they are characterized by different interatomic distances with neighboring atoms, different values for the charge localized on the atom and of the energy characteristics of chemisorption (Table 2). Comparison of $E_{a c t}=0.40-0.78 \mathrm{eV}$ for a hexagonal graphene-like structure on the surface with a BV-complex (defect 2) with the literature data [11, 12] suggests that the chemisorption of hydrogen will primarily occur on the $\mathrm{C} 1, \mathrm{C} 2$ and $\mathrm{C} 4$ atoms of the "hexagon", for which the 
TABLE 2. The geometry and energy characteristics of a hydrogen atom adsorption on the diamond surface containing a "boron + vacancy" complex

\begin{tabular}{|c|c|c|c|c|c|c|}
\hline \multirow{2}{*}{ Surface atom } & \multicolumn{2}{|c|}{$E_{a c t}(\mathrm{eV})$} & \multicolumn{2}{c|}{ Heat of adsorption $q(\mathrm{eV})$} & \multicolumn{2}{c|}{ C (B) - H distance $d, \AA$} \\
\cline { 2 - 7 } & Defect 1 & Defect 2 & Defect 1 & Defect 2 & Defect 1 & Defect 2 \\
\hline \hline C1 & 0.85 & 0.43 & 3.18 & 2.74 & 1.102 & 1.115 \\
\hline C2 & 0.85 & 0.41 & 3.24 & 3.54 & 1.103 & 1.110 \\
\hline C3 & 1.10 & 0.64 & 2.64 & 2.43 & 1.116 & 1.134 \\
\hline C4 & 0.86 & 0.40 & 3.23 & 3.60 & 1.103 & 1.109 \\
\hline C5 (B) & 0.86 & 0.52 & 3.20 & 2.98 & 1.118 & 1.160 \\
\hline C6 & 1.11 & 0.78 & 2.72 & 3.22 & 1.124 & 1.125 \\
\hline
\end{tabular}

activation energy of the first hydrogen atom adsorption is $E_{a c t}=0.40-0.43 \mathrm{eV}$, and only next on the dimer atoms in the ordered surface areas $\left(E_{a c t}=0.65 \mathrm{eV}\right)$.

Thus, BV-complexes in the near-surface layers of $\mathrm{C}(100)-(2 \times 1)$ diamond lead to a significant change in the geometry and electronic state of the surface: there appear some active centers of chemisorption that affect the mechanism and energy of the whole process.

\section{Acknowledgements}

The work was supported by the Ministry of Education and Science of the Russian Federation, scientific project \#14.580.21.0003 (RFMEFI58015X0003).

\section{References}

[1] Kraft A. Doped diamond: a compact review on a new, versatile electrode material. Int. J. Electrochem. Sci., 2007, 2, P. 355-385.

[2] Goss J.P., Briddon P.R. Theory of boron aggregates in diamond: First-principles calculations. Phys. Rev. B, $2006,73,085204$.

[3] Kunisaki A., Muruganathan M., Mizuta H., Kodera T. First-principles calculation of a negatively charged boron-vacancy center in diamond. Jpn. J. Appl. Phys., 2017, 56, 04CK02.

[4] Stallcup II R.E., Villarreal L.M., et al. Atomic structure of the diamond (100) surface studied using scanning tunneling microscopy. J. Vac. Sci. Technol. B, 1996, 14, P. 929-932.

[5] Ristein J. Surface science of diamond: Familiar and amazing. Surf. Sci., 2006, 600, P. 3677-3589.

[6] Stewart J.J.P. MOPAC2016, Version 16.158W, Stewart Computational Chemistry, URL: http: //OpenMOPAC.net/.

[7] Lvova N.A., Ananina O.Yu. Quantum chemical simulations of water adsorption on a diamond (100) surface with vacancy defects. Rus. J. Phys. Chem. A, 2013, 87 (9), P. 1515-1519.

[8] Lvova N.A., Ponomarev O.V., Ananina O.Yu., Ryazanova A.I. Boron atoms in the diamond subsurface layers: quantum-chemical modeling. Rus. J. Phys. Chem. A, 2017, 91 (8), P. 1320-1325.

[9] Butrimov P.A., Anan'ina O.Yu., Yanovskii A.S. Quantum-chemical study of interaction of hydrogen atoms with graphene. J. Surf. Investigation, X-ray, Synchrotron Neutron Techniques, 2010, 4 (3), P. 476-479.

[10] Lvova N.., Ananina .Yu. Theoretical study of graphene functionalization by F- and FHF- ions from associates with water molecules. Comp. Mater. Sci., 2015, 101, P. 287-292.

[11] Hukka T.I., Pakkanen T.A., D’Evelin M.P. Chemisorption of hydrogen on the diamond (100) $2 \times 1$ surface: an ab initio study. J. Phys. Chem., 1994, 98, P. 12420-12430.

[12] Filicheva Yu.A., Lvova N.A., Ananina .Yu. Quantum-chemical simulation of interaction of hydrogen with diamond nanoclusters. Fuller. Nanotub. Car. N., 2012, 20, P. 616-621. 